\title{
Adjunction and Branchingness Effects in Syntax-Prosody Mapping
}

\author{
Jennifer Bellik \& Nick Kalivoda \\ University of California, Santa Cruz.
}

\section{Introduction}

Several current approaches to the syntax-prosody interface employ constraints that enforce mapping between syntactic structure and prosodic structure, demanding that syntactic XPs correspond to phonological phrases, and vice versa. For example, Match Theory (MT), an approach to the mapping from syntactic to prosodic structure couched within Optimality Theory (Selkirk 2011, Elfner 2012, Myrberg 2013), predicts that prosodic structure should closely resemble syntactic structure, and that deviations from perfect syntaxprosody isomorphism should only arise due to markedness constraints. Similarly, Align/Wrap Theory (Truckenbrodt 1995, 1999), an OT implementation of Selkirk's (1986) End-Based Approach, employs alignment constraints which demand that the left and right edges of syntactic XPs map to corresponding left and right edges of phonological phrases, as well as a constraint WRAP-XP, which requires every XP to be enclosed in a corresponding phonological phrase in the prosodic parse.

While what counts as a visible "XP" for the mapping constraints is usually straightforward, a question arises in cases of adjunction. Given the segment theory of adjunction (May 1985, Chomsky 1986, Truckenbrodt 1999), the syntax-prosody interface theorist must determine which segments of a polysegmental category are "visible" to mapping constraints: the lowest, the highest, or all segments.

(1) Adjunction Visibility Settings
a. $H+L$ visible
b. Only H visible
c. Only L visible
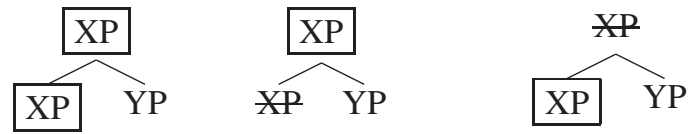

Following Truckenbrodt (1995, 1999), Selkirk (2011:483, fn. 38) suggests that (1a) is the correct treatment of such structures: only the lowest segment of XP is visible to the Match constraints. However, there is no a priori reason to think that higher segments of XP should not be visible to mapping constraints. We conduct a study of the prosody of adjunction structures, manipulating the sensitivity of the mapping constraints to the high vs. low segments of the adjunction structure XPs. We find that under some visibility settings, both Align/Wrap and Match Theory make a surprising prediction regarding the prosodic phrasing of adjunction structures: that adjuncts will sometimes phrase prosodically with a linearly adjacent but syntactically distant word, to the exclusion of their syntactically closer host. For example, an adjective may phrase with the verb to its right in a $\phi$ that excludes the noun modified by the adjective. In short, adjuncts are predicted to sometimes fail to cohere with their hosts: the Adjunct Non-Cohesion Prediction (ANCP).

(2) Adjunction Non-Cohesion Prediction

$$
[\mathrm{TP}[\mathrm{NP} \mathrm{N} \text { A }][v \mathrm{P} \mathrm{V} \ldots]] \rightarrow \ldots \mathrm{N} \ldots(\phi \ldots \mathrm{A} \ldots \mathrm{V} \ldots) \ldots
$$

The systems we consider predict two varieties languages with adjunct/host non-cohesion: languages where non-cohesion ties with cohesion, and languages where non-cohesion candidates win. To our

\footnotetext{
* We would like to thank Junko Itô, Armin Mester, Alan Prince, Ozan Bellik, and the participants of the UCSC 2015 syntax-prosody seminar for helpful comments and discussion. All errors are our own. This material is based upon work supported by the National Science Foundation Graduate Research Fellowship Program under Grant No. DGE-1339067.
}

(C) 2016 Jennifer Bellik \& Nick Kalivoda

Proceedings of AMP 2015

Completed February 2, 2016 
knowledge, no such languages exist. That is, in the languages we know of whose word order creates opportunities for phrasings like $\left(_{\phi} \mathrm{N}\left({ }_{\phi} \mathrm{A} V\right)\right)$, the non-cohesive phrasing is unattested in the literature, both as an option and as a preferred phrasing. These languages include French, Italian, and Spanish (Nespor \& Vogel 1986), and Kinyambo (Bickmore 1989, 1990), the language used as our case study in this paper. Thus, the ANCP may in fact be a pathological prediction (at least at the phrasal level), not just a surprising one. This study explores what axioms in a prosodic theory result in the ANCP, so that if the ANCP turns out to be a pathological prediction, we will know what settings to use in the syntax-prosody interface theory in order to avoid the pathology; and conversely, if a language that fulfills the ANCP is found, then we will know what settings to use so that our theory correctly generates that language as part of the typology.

An additional goal of this investigation is to determine whether Match Theory provides the empirical coverage that Align/Wrap Theory does. Specifically, MT is claimed to avoid some of Align/Wrap's putative overgeneration problems (Selkirk 2011). But might MT undergenerate in its quest to avoid overgeneration? In particular, does MT predict the well-known branchingness effects seen in Kinyambo prosody, documented in Bickmore (1990)? To this end we also explore two different formulations of binarity constraints: one sensitive to the number of prosodic words a node dominates, another sensitive to the number of children a node has.

Our investigation employs the computational tools of SPOT, a JavaScript application we have developed for OT experiments on the syntax-prosody interface, and OTWorkplace (Prince, Tesar, \& Merchant 2015), an Excel-based tool for calculating typologies and crucial rankings. We find, first, that making high XP segments in adjunction structures visible has beneficial effects on the typology; second, that Match Theory is able to avoid adjunct non-cohesion when high XP segments are visible to Match, but that Align/Wrap is still subject to the ANCP under all visibility settings; and third, that branchingness effects in Kinyambo can be captured in MT under either version of the binarity constraints that we consider.

The structure of the paper is as follows. $\S 2$ presents the language data from Kinyambo that we employ in this case study. $\S 3$ describes the motivations, tools and factors in our computational experiment. In $\S 4$, we present our main results. $\S 5$ concludes.

\section{Language data}

Bickmore (1990) reports on a phonological process in Kinyambo which is prosodically interesting in that it clearly delimits phonological phrases. These phrases do not simply correspond to syntactic phrases, but are sensitive to phonological size. The language provides useful data for examining the phrasing of adjuncts, since APs are post-nominal and subjects are preverbal, allowing us to examine the syntactic configuration [NOUN ADJECTIVE] [VERB] — exactly the configuration under which we expect adjunct-host cohesion, and in which a particular constraint ranking might predict adjunct non-cohesion.

The process we consider is that of High Tone Deletion (HTD), as reported by Bickmore (1990). Words that underlyingly bear a high tone are underlined.

(3) High Tone Deletion (Bickmore 1990)

$$
\mathrm{H} \rightarrow \emptyset /(\phi \ldots(\omega \ldots \ldots)(\omega \ldots \mathrm{H} \ldots) \ldots)
$$

When a phonological phrase in Kinyambo ends with a word bearing a high tone, any high tones on the nonphrase-final words are deleted. For instance, although abakózi 'workers' pronounced in isolation bears a penultimate high tone, the same word to the left of a verb (4a) or adjective (4b) surfaces without this tone.

(4) a. (ф abakozi bákajúna) workers helped

'The workers helped.'

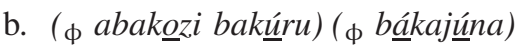

workers mature helped

'The mature workers helped.'

Bickmore (1990) shows that in simple intransitives, one-word NPs phrase with V, as in (4a). When the subject NP contains more than two words, however, it phrases apart from the following verb. This is shown by the lack of high tone deletion on the postnominal adjective bakúru 'mature' in (4b). If all three words 
in the sentence were phrased together, only the verb would bear high tone. The fact that there is still no high tone on abakozi 'workers' in (4b) shows that the noun forms a $\phi$ with the adjective, whose faithfully surfacing high tone induces deletion of that on the noun.

If adjectives in subjects failed to prosodically cohere to their syntactic host, but instead formed a phonological phrase with the verb, the result in a hypothetical language Kinyambo' would be the following.

(5) Adjunct non-cohering form: HTD on adjective, not on noun

*(ф abakózi) (ф baküru bákajúna)

workers mature helped

Here, given HTD, the adjective would lose its underlying high tone, but the noun would not. While this is not the right result for actual Kinyambo, it is worth asking whether a given theory of syntax-phonology mapping might predict a language like Kinyambo' to exist. We hypothesize that it should not, though we leave the question open. But even without settling the question empirically, we can examine factorial typologies of well defined optimality-theoretic systems (in the sense of Alber, DelBusso, \& Prince 2015) to determine whether a theory predicts a language with the phrasing in (5) to be possible.

In order to further investigate the phrasing of adjuncts, we examine an additional pair of sentences from Bickmore (1990): a double object construction with a pro subject, as well as a structurally minimally contrasting clause with a right-adjoined adverbial, shown in $(6 \mathrm{a}-\mathrm{b})$, respectively.

(6) (ф nejákuh’ omutáhi ebitóoke) he-will-give friend bananas

'He will give the friend bananas.'

(ф nejákuh' ómukoz’ ékitebe mpóra) he-will-give worker chair slowly

'He will give the worker a chair slowly.'

Assuming that mpóra 'slowly' is right-adjoined to $v \mathrm{P}$, this pair provides an additional criterion by which to evaluate various theories of the syntax-phonology mapping, particular with regards to the interpretation of adjunction structures. Note that although certain $\phi$-medial words in (6) bear surface high tones, these are morphologically required and thereby exempt from HTD; high tone deletion has in fact occurred throughout. The reader is referred to Bickmore $(1989,1990)$ for details.

\section{Methods}

To draw valid conclusions regarding an optimality-theoretic system, we need a precise definition of both GEN, the candidate generation function, and CON, the constraint set. In our investigations of the treatment of adjunction structures in syntax-prosody mapping, we employ the candidate generator described in $\S 3.1$ and the constraints in $\S 3.2$.

3.1 Candidate generation We use the computational tool SPOT (Bellik, Bellik, \& Kalivoda 2016) to generated candidate sets consisting of every licit prosodic parse (within a particular theory of the prosodic hierarchy) for an input string of words, the terminal string of a well-formed syntactic tree. What counts as a "licit prosodic parse" is detailed in (8).

(8) A prosodic tree $P$ is an output of $\operatorname{GEN}(T)$, where $T$ is a string of terminals, iff:

a. $P$ is rooted in a node of category $\mathrm{l}$,

b. the string of terminals in $P, P(T)$, is equal to $T$,

c. every terminal in $P(T)$ is of category $\omega$,

d. every node in $P$ that is neither a root nor a terminal node is of category $\phi$,

e. $P$ contains no node $x$ of category $\phi$ such that $x$ dominates exactly one nonterminal node

The theory of the prosodic hierarchy we employ here is that of Selkirk (2011), Itô \& Mester (2013), and others. As in the theory developed by Nespor \& Vogel (1986), prosodic categories include the intonational 
phrase $\iota$, the phonological phrase $\phi$, and the phonological word $\omega$. Unlike Nespor \& Vogel (1986), we exclude the utterance and the clitic group as prosodic categories, and adopt a form of Weak Layering which allows for recursive prosodic categories, level-skipping, and exocentric constituents. We abstract away from all lower-level categories (foot, syllable, etc.), treating morphosyntactic and phonological words as atomic in order to focus on phrase-level phenomena.

3.2 Design We have generated twelve typologies resulting from a $3 \times 2 \times 2$ manipulation, where the factors were high/low XP segment visibility to mapping constraints (high+low segments visible, only high segment visible, only low segment visible); mapping constraints (Match vs. Align/Wrap); and the precise formulation of binarity constraints (binarity by branchingness vs. binarity by words).

All Match systems employ the following mapping constraints, adopted from Selkirk (2011) and Elfner (2012).

(9) $\operatorname{MATCH}(\phi, X P)$

Assign a violation for every node $p$ of category $\phi$ in the prosodic tree for which there is no node $s$ in the syntactic tree such that every terminal node dominated by $p$ corresponds to a terminal node dominated by $s$, and vice versa.

(10) $\operatorname{MATCH}(\mathrm{XP}, \phi)$

Assign a violation for every node $s$ of category XP in the syntactic tree for which there is no node $p$ of category $\phi$ in the prosodic tree such that every terminal node dominated by $s$ corresponds to a terminal node dominated by $p$, and vice versa.

The Match constraints do not refer to a particular edge (left or right) of a the XP or phonological phrase. Instead, they demand that a phrase and its correspondent share exactly the same terminal material.

Meanwhile, all Align/Wrap systems use the mapping constraints Align(XP, L, $\phi$, L), Align(XP, R, $\phi$, R), and WRAP(XP) (Truckenbrodt 1995, 1999). The alignment constraints follow the schema of Generalized Alignment (McCarthy \& Prince 1993).

(11) $\operatorname{Align}(\mathrm{XP}, \delta, \phi, \delta)$

Assign a violation for every node $s$ of category XP such that there is no node $p$ of category $p h i$ such that the $\delta$ edge of $s$ corresponds to the $\delta$ edge of $p$, where $\delta$ is Left or Right.

(12) WraP(XP)

Assign a violation for every node $s$ of category XP such that there is no node $p$ of category $\phi$ such that every terminal dominated by $s$ has a corresponding terminal dominated by $p$.

The alignment constraints schematized in (11) differ crucially from Match constraints in referring only to a particular edge of each phrase. WRAP-XP, by contrast, says nothing about phrase edges, but insists that all terminals of some XP be phrased together in the prosodic tree, though not necessarily to the exclusion of additional material.

For both Match and Align/Wrap, we define correspondence as follows.

\section{(13) Terminal Node Correspondence}

A prosodic terminal $p$ and a syntactic terminal $s$ correspond to each other iff $p$ matches the phonological content of the lexical entry for $s$. (Note that in our systems all terminals are words.)

The mapping constraints shown above are responsible for the fact that syntactic and prosodic structure are related, with syntactic constituents often corresponding to prosodic constituents and vice versa. However, as we have seen in $\S 2$, prosodic structure may deviate from syntactic sturcture. In Kinyambo (Bickmore 1990), as in many other languages (see Nespor \& Vogel 1986, Elfner 2012), the number of elements contained in a constituent can play a crucial role in phrasing, meaning that syntactic non-terminals themselves are not the sole determinant of phrasing.

In each system we explore, we employ two binarity constraints: one in the family BINMIN, requiring $\phi s$ to be minimally binary, and one in the family BINMAX, militating against $\phi$ s with more than two designated elements. An important question, though, regards what elements count as contributing to the size of a $\phi$. We explore two possibilities: first, that the number of nodes immediately dominated by a $\phi$ gives the relevant 
count for the binarity constraints, and second, that the number of phonological words $\omega$ dominated by a $\phi$ (not necessarily immediately dominated) is what is relevant. The relevant BINMAX constraints are defined in (14).

\section{(14) a. Binarity by branchingness}

$\operatorname{BINMAX}(\phi, \mathrm{BR}):$ Assign a violation for every node $p$ of category $\phi$ such that $p$ has more than 2 children (i.e. more than two branches).

b. Binarity by words

$\operatorname{BINMAX}(\phi, \omega)$ : Assign a violation for every node $p$ of category $\phi$ such that $p$ dominates more than 2 terminals (phonological words).

Each of our systems has a particular setting for "visibility" in terms of XP-adjunction. In HL systems, both the high and low segment of an adjunct's host count for the mapping constraints; in H systems, only the highest segment counts; and in L systems, only the lowest counts. The three possibilities are illustrated in (15).

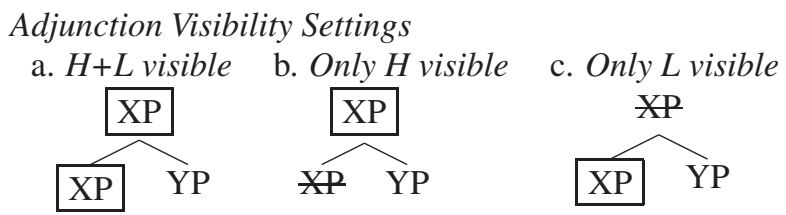

In all conditions, $\mathrm{AP}, \mathrm{AdvP}$, and functional projections ( $\mathrm{TP}, v \mathrm{P}, v \mathrm{P}$ headed by trace) count for mapping. Note that these assumptions are usual for Match Theory but not for Align/Wrap; work on Align/Wrap generally assumes Truckenbrodt's (1995, 1999) Lexical Category Condition (LCC), which renders functional projections and projections headed by a trace invisible. A closer evaluation of the consequences of Align/Wrap will require adopting the LCC in additional systems. However, for now we keep these visibility settings constant across conditions in order to more clearly see the results of the factors under investigation.

Finally, a particular constraint in CON was held constant in all systems: EQUALSISTERS (Myrberg 2013), a constraint demanding that sisters in a prosodic tree be of the same category of the prosodic hierarchy. We have implemented this constraint in several different ways in SPOT, each with subtle and interesting differences, but in the present study, EQUALSISTERS assigns one violation for every pair of adjacent sister nodes in a prosodic tree of different categories.

3.3 Design summary To summarize, details of the twelve systems we investigate are provided in (16).

\section{(16) Factors}

a. Visibility:

In 'HL' systems, all segments of XP are visible; in ' $\mathrm{H}$ ' systems, only the highest segment is; in ' $\mathrm{L}$ ' systems, only the lowest segment is.

b. Mapping constraints:

For Match systems, Con includes $\operatorname{MATCH}(\mathrm{XP}, \phi)$ and $\operatorname{MATCH}(\phi, \mathrm{XP})$; for Align/Wrap systems, Con includes Align(XP, R, $\phi, R), \operatorname{Align}(X P, L, \phi, L)$, and WraP(XP).

c. Binarity constraints:

In 'BinBr' systems, binarity constraints count nodes' children. In 'BinWd' systems, they count the number of $\omega$ s dominated by a node.

3.4 Computational tools for theory comparison We employ the computational tools OT Workplace (Prince, Tesar, \& Merchant 2015) and SPOT (Bellik, Bellik, \& Kalivoda 2015), a new JavaScript application which we have developed. Syntax-Prosody in Optimality Theory JavaScript Application (henceforth SPOT) is a tool developed by Jennifer Bellik, Ozan Bellik, and Nick Kalivoda, and intended for use with OT Workplace (Prince, Tesar, \& Merchant 2015). SPOT's function is twofold: first, to exhaustively generate all the possible prosodic parses of a string of words (candidate generation); and second, to automatically evaluate their violation counts for various constraints (constraint evaluation). To our knowledge, this is the only tool that automates these functions. 
In an OT investigation, all possible candidates must be considered in order to draw valid conclusions about which candidate is selected as optimal by the constraint set being used. It is difficult and timeconsuming to manually generate all possible candidates, because their number can be so large. As soon as an investigation begins considering phrases with more than a few words, the number of prosodic parses becomes unmanageable. For example, in the realm of syntax-prosody mappings, a string of two words has 8 possible prosodic parses (GEN settings: recursivity allowed, non-exhaustive parsing allowed, headedness not enforced); a string of 3 words, 48 prosodic parses; a string of 4 words, 352 parses; and so on. Moreover, manually evaluating the number of violations that each candidate incurs becomes burdensome and errorprone when so many candidates are considered. Therefore, it is vital to automate both candidate generation and constraint evaluation.

OTWorkplace (Prince, Tesar, \& Merchant 2015; henceforth OTW) provides tools for candidate generation and constraint evaluation. However, these tools are less suited for syntax-prosody investigations, because of their restricted scope. OTW's candidate generation is confined to metrical parsing, and therefore cannot generate the highly articulated prosodic trees needed for a syntax-prosody investigation. Similarly, OTW's automated constraint evaluation relies on regular expressions, which are too low on the Chomsky Hierarchy of formal languages to cope with trees (Chomsky 1959). Consequently, a new tool for candidate generation and constraint evaluation is needed for more rigorous OT investigations of the syntax-prosody mapping.

We have developed SPOT to be such a tool. SPOT is implemented in JavaScript, which enables easy representation of trees of any level of complexity, as well as convenient interface with html and the World Wide Web. A limited html interface is currently available online. SPOT provides both exhaustive generation of the prosodic candidates, and automatic evaluation of the violation profiles of each candidate.

3.5 Syntactic input Four syntactic trees were held constant as inputs to the twelve OT systems developed. Representations of these syntactic trees in JavaScript were used for automated constraint evaluation. The first two forms, (17) and (18), were selected since they minimally contrast in the presence of an adjoined AP in the second, but not in the first. Similarly, the trees in (19) and (20), each containing a double object construction, contrast in the presence or absence of a sentence-final adverb, right-adjoined to $v \mathrm{P}$.

Various nodes in the trees below are labeled as phrasal, but consist of only a single word. Adopting the Bare Phrase Structure of Chomsky (1995), we assume that any phrase that does not project further is counted as a maximal projection by the syntax-phonology mapping constraints. Thus, the one-word subject abakozi 'workers' in (17) counts as a phrase for the various mapping constraints we employ.

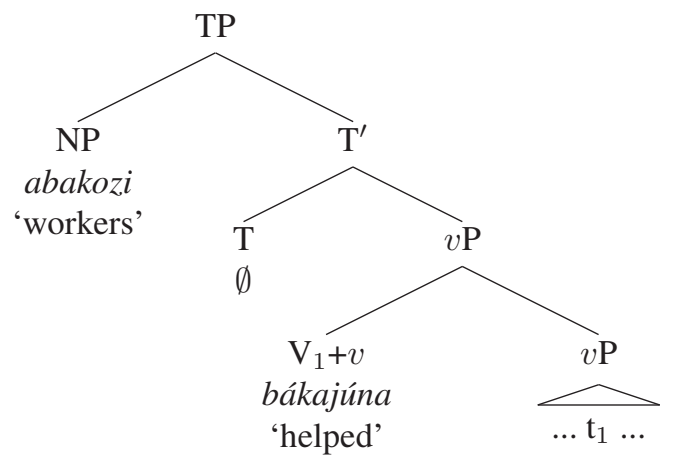




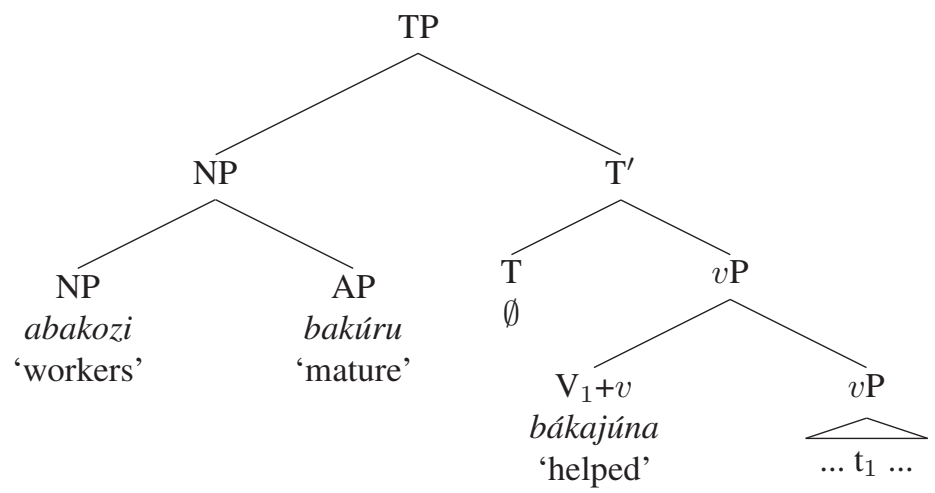

(19)

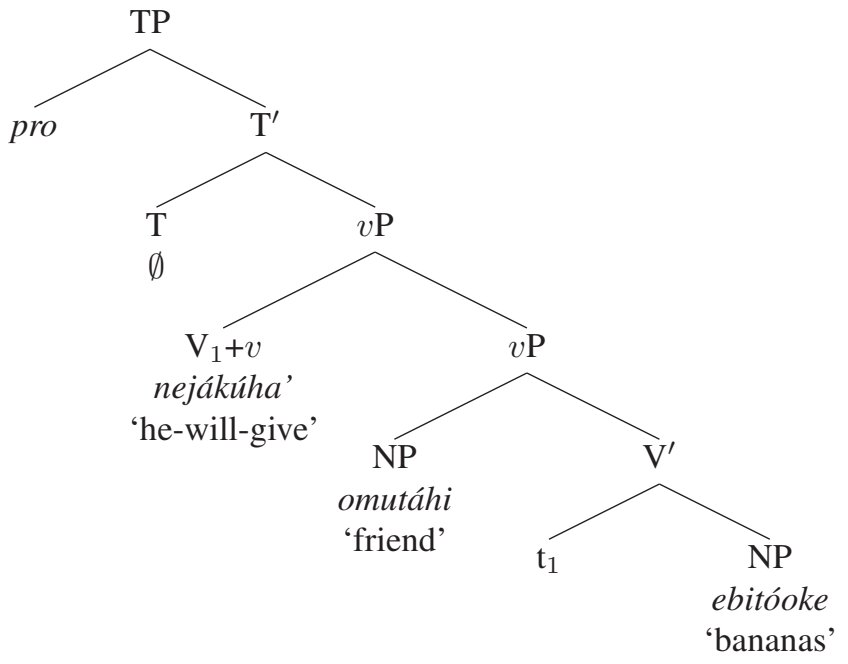

(20)



The syntactic representations given above are contemporary adaptations of those proposed by Bickmore (1990). 


\section{Results}

4.1 Binarity constraints The choice between word-based and branchingness-based binarity constraints did not have any effect on a system's successfully generating phrasings consistent with those found in Kinyambo, nor did it have any effect on whether a system predicts Adjunct Non-Cohesion or not. The choice does, however, affect typology size. Generally, typologies in systems with branchingness-based binarity are smaller than those with word-based binarity constraints. In the remainder of the paper, the discussion focuses on branchingness binarity systems and leaves aside the word binarity systems.

4.2 Phrasing in Kinyambo In terms of empirical coverage, we find that all four HL+Match and $\mathrm{H}+$ Match systems, i.e. all systems involving Match constraints in which the highest segment of a syntactic $\mathrm{XP}$ is visible, succeed in generating phrasings consistent with High Tone Deletion from Kinyambo (Bickmore 1990). Furthermore, none of the eight additional systems we tested had the same success; each fell short on at least one of the four input sentences.

The following two tableaux partially illustrate the success of the Match systems in which the highest segment of XP is visible to the mapping constraints. Recall that this means that in the subject [NP NP AP] in (22), all three nodes (AP, the low NP segment, and the high NP segment) count for МATCH-XP and MAтсH- $\phi$. The tableaux show ranking conditions within the system HL+Match+Br, one of the four successful systems. Each tableau includes all non-harmonically bounded candidates within the system-i.e., all candidates which surface as optimal in some language in the factorial typology, as determined using SPOT and OTWorkplace. ${ }^{1}$

\section{(21) Successful phrasing of Kinyambo two-word intransitive in $H L+M a t c h+B r$}

\begin{tabular}{|c|c|c|c|c|c|}
\hline$[\mathrm{TP}[\mathrm{NP} N][\mathrm{vP} \mathrm{V}]]$ & BINMAX & EQSIS & BINMIN & MATCH-XP & MATCH- $\phi$ \\
\hline "a. $\quad(\mathrm{N} \mathrm{V})$ & & & & $\mathrm{NP}, \mathrm{vP}$ & \\
\hline$((\mathrm{N})(\mathrm{V}))$ & & & $\overline{\mathrm{W}_{2}}$ & $\overline{\mathrm{L}_{0}}$ & \\
\hline
\end{tabular}

The tableau in (21) shows single elementary ranking condition (ERC) that arises from pitting the flat (actual Kinyambo) phrasing in (a) against its lone competitor, in which both the NP and $v \mathrm{P}$ are matched with their own $\phi s$. While candidate (b) bests (a) in terms of MATCH-XP, higher ranking BINMIN forces the less faithful and less marked phrasing in (a). We therefore preserve Bickmore's (1990) insight, essentially implementing his mapping principles (including the binarity-imperative for $\phi s$ ) as violable constraints.

A similar state of affairs holds in the three-word intransitive in (22), where the fact that the subject is branching (i.e. contains two words) makes it possible to phrase it on its own, apart from the verb.

\section{Successful phrasing of Kinyambo three-word intransitive in $\mathrm{HL}+\mathrm{Match}+\mathrm{Br}$}

\begin{tabular}{|c|c|c|c|c|c|c|}
\hline$[\mathrm{TP}[\mathrm{s}$ & $\mathrm{NP} N][$ [AP A]][ [vP V] & BINMAX & EQSIS & BINMIN & MATCH-XP & МАТСH- $\phi$ \\
\hline a. & $((\mathrm{N} A)(\mathrm{V}))$ & & & 1 & $\mathrm{NP}, \mathrm{AP}$ & \\
\hline b. & $\overline{(((\mathrm{N})(\mathrm{A}))(\mathrm{V}))}$ & & & $\mathrm{W}_{3}$ & $\mathrm{~L}_{0}$ & \\
\hline c. & $((\mathrm{N} \mathrm{A}) \mathrm{V})$ & & $\mathrm{W}_{1}$ & $\mathrm{~L}_{0}$ & $\mathrm{~W}_{\mathrm{NP}, \mathrm{AP}, \mathrm{vP}}$ & \\
\hline d. & $(\mathrm{N} \mathrm{A} \mathrm{V})$ & $\mathrm{W}_{1}$ & & $\mathrm{~L}_{0}$ & $\mathrm{~W}_{\mathrm{NP}, \mathrm{NP}, \mathrm{AP}, \mathrm{vP}}$ & \\
\hline
\end{tabular}

The optimal candidate (22a) violates BINMIN once, due to the one-word $\left({ }_{\phi} \mathrm{V}\right)$, and fails to match the lower segment of NP and the entire AP to $\phi s$. The additional markedness constraints BINMAX and EQUALSISTERS now come into play, as the ERCs from tableau-rows (c) and (d) illustrate. BINMAX rules out the ternarybranching flat phrasing in (d), which might have been expected given the flat phrasing of the non-branching subject sentence, and EQUALSISTERS rules out a candidate similar to the winner but lacking a $\phi$ for the verb. And, as in (21), BINMIN crucially outranks MATCH-XP, favoring a flat subject-internal phrasing as in (a) over the highly articulated (b), which in Kinyambo would wrongfully prevent high tone deletion from applying anywhere.

1 Here and in all additional tableaux, cells which would contain only ' 0 ' or ' $\mathrm{e}_{0}$ ' are left blank for readability. ' $\mathrm{L}_{0}$ ' cells are included, as these are crucial for establishing ERCs. 
This subsection has shown that a Match theoretic system is capable of dealing with the facts from a small fragment of Kinyambo. The same systems are also able to yield the correct phrasing for the ditransitives provided in $\S 2$, though we exclude tableaux here for reasons of space. However, it is important to note that this success is due in part to the visibility of the highest segment of NP in the structure [NP NP AP], contrary to what has been proposed elsewhere for other languages (Selkirk 2011, Truckenbrodt 1995, 1999). Making the top segment of the maximal projection invisible to the mapping constraints causes major problems, to which we now turn.

4.3 Visibility settings Visibility settings have a significant effect on typology, and interact with the mapping settings. High XP segment visibility eliminates adjunct non-cohesion from the typologies of all Match systems, but contributes adjunct non-cohesion winners to the typologies of Align systems. The Lowonly visibility systems will be discussed first. Whenever the higher segment of the XP adjunction structure is invisible to mapping constraints (that is, in the Low-only visibility setting), there are two effects: (i) Kinyambo does not appear in the typology (a problem of undergeneration), and (ii) the typology contains languages in which Adjunct Non-Cohesion candidates tie with Adjunct Cohesion candidates. Non-cohesion ties appear in Low-only Match systems, and in all Align/Wrap systems. This is illustrated in (23) with a Match system.

\section{Adjunct Cohesion tie in $\mathrm{L}+\mathrm{Match}+\mathrm{Br}$}

\begin{tabular}{|l||c|c|c|c|c|}
\hline$[$ TP [NP [NP N] [AP A]] [vP V]] & MATCH-XP & MATCH- $\phi$ & ES & BINMIN & BINMAX \\
\hline \hline a. $((\mathrm{N} \mathrm{A})(\mathrm{V}))$ & $\mathrm{NP}, \mathrm{AP}$ & $\left(_{\phi} \mathrm{NA}\right)$ & & 1 & \\
\hline b. $((\mathrm{N})(\mathrm{A} \mathrm{V}))$ & $\mathrm{e}_{\mathrm{AP}, \mathrm{vP}}$ & $\left.\mathrm{e}_{\left(_{\phi}\right.} \mathrm{AV}\right)$ & & $\mathrm{e}_{1}$ & \\
\hline
\end{tabular}

In (23), the actually attested phrasing in which the two-word subject phrases separately from the verb ties on every constraint with the Adjunct Non-Cohering candidate (b). Each candidate violates MATCH-XP twice: candidate (a) for failing to match the low NP segment and the AP, and candidate (b) for failing to match the AP and $v \mathrm{P}$. The fact that candidate (a) phrases the entire subject NP as a single $\phi$ is irrelevant since the highest segment of NP is invisible in this system. Similarly, (a) and (b) somewhat counterintuitively tie in terms of MATCH- $\phi$. Candidate (b) violates MATCH- $\phi$ once since the phrase ( $\phi$ AV) has no syntactic correspondent. Candidate (a) fares no better; although the phrase $\left({ }_{\phi}\right.$ NA) does have a syntactic correspondent, namely the high segment of NP, that correspondent is not visible in this system, meaning that the constraint cannot "see" the relevant node and assigns a violation. And being essentially mirror images of one another, neither candidate outperforms the other in terms of the three markedness constraints. The tie cannot be resolved unless the highest segment of each maximal projection is made visible.

When the High XP segment is made visible to mapping constraints (High and High+Low visibility settings), different effects obtain in the Match systems compared to the Align systems. Match systems where the high segment is visible avoid the undergeneration problem - they all contain at least one language with phrasings compatible with the Kinyambo data. Moreover, they do not allow any adjunct non-cohesion, whether as ties or as winners. In Align systems where the high XP segment is visible, however, non-cohesion winners appear in the typology, but no Kinyambo-compatible language is in the typology; the undergeneration problem persists.

4.4 Impact of mapping constraints. As discussed above, the choice between Match and Align/Wrap for the mapping constraints interacts with the visibility settings. However, some generalizations can be made about all Align systems in our experiment. All six Align/Wrap systems have an undergeneration problemtheir typologies predict the phrasing of ( $\phi$ VERB NOUN NOUN ADVERB) that is attested in Kinyambo to be impossible. Moreover, every Align/Wrap system contains in its typology a language or languages where adjunct non-cohesion emerges as co-optimal (if only the Low XP segment is visible), or optimal (if the High $\mathrm{XP}$ segment is visible). 
ANC candidate can win in Align/Wrap systems

\begin{tabular}{|c|c|c|c|c|c|c|c|}
\hline [TP [- & $\left.\mathrm{NP} \mathrm{N}][\mathrm{AP} \mathrm{A}]]\left[v_{\mathrm{P}} \mathrm{V}\right]\right]$ & WRAP & BMAX & BMIN & AL-L & AL-R & EQSIS \\
\hline a. & $(\mathrm{N}(\mathrm{A} \mathrm{V}))$ & & & & $v \mathrm{P}$ & NP, AP & . \\
\hline b. & $((\mathrm{N} A) \mathrm{V})$ & & & & $\mathrm{W}_{\mathrm{AP}, v \mathrm{P}}$ & $\mathrm{L}_{\mathrm{NP}}$ & $\mathrm{e}_{1}$ \\
\hline c. & $(\mathrm{N}(\mathrm{A}(\mathrm{V})))$ & & & $\mathrm{W}_{1}$ & $\mathrm{~L}_{0}$ & $\mathrm{e}_{\mathrm{NP}, \mathrm{AP}}$ & $\mathrm{e}_{1}$ \\
\hline d. & $(((\mathrm{N}) \mathrm{A}) \mathrm{V})$ & & & $\mathrm{W}_{1}$ & $\mathrm{~W}_{\mathrm{AP}, v \mathrm{P}}$ & $\mathrm{L}_{0}$ & $\mathrm{~W}_{2}$ \\
\hline e. & $((\mathrm{N})((\mathrm{A})(\mathrm{V})))$ & & & $\mathrm{W}_{3}$ & $\mathrm{~L}_{0}$ & $\mathrm{~L}_{0}$ & $\overline{\mathrm{W}_{2}}$ \\
\hline f. & $(((\mathrm{N})(\mathrm{A}))(\mathrm{V}))$ & & & $\mathrm{W}_{3}$ & $\mathrm{~L}_{0}$ & $\mathrm{~L}_{0}$ & $\mathrm{~L}_{0}$ \\
\hline g. & $((\mathrm{N})(\mathrm{A}$ V) $)$ & & & $\overline{\mathrm{W}_{1}}$ & $\mathrm{~L}_{0}$ & $\mathrm{~L}_{\mathrm{AP}}$ & $\mathrm{L}_{0}$ \\
\hline h. & $((\mathrm{N} A)(\mathrm{V}))$ & & & $\overline{W_{1}}$ & $\mathrm{e}_{v \mathrm{P}}$ & $\mathrm{L}_{\mathrm{NP}}$ & $\mathrm{L}_{0}$ \\
\hline i. & (N A V) & & $\mathrm{W}_{1}$ & & $\mathrm{~W}_{\mathrm{AP}, v \mathrm{P}}$ & $\mathrm{e}_{\mathrm{NP}, \mathrm{AP}}$ & $\mathrm{L}_{0}$ \\
\hline
\end{tabular}

The tableau in (24) includes all possible optima within the system L+Align+BinBr. When ranked higher than the alignment constraints and EQUALSISTERS, the binarity constraints BINMAX( $\phi$,branches) and $\operatorname{BINMIN}(\phi$, branches) rule out all candidates in which a $\phi$ is not strictly binary-branching, leaving only (24a) and (24b) as possibilities. These two candidates tie in terms of EQUALSISTERS, each violating this constraint exactly once: the former due to the configuration $\left[\omega_{\mathrm{N}} \phi\right]$, the latter due to $\left[\phi \omega_{\mathrm{V}}\right]$. The decision is therefore left to the relative ranking of ALIGN-L and ALIGN-R.

Both (24a) and (24b) successfully align the right edge of $v \mathrm{P}$ (since $\mathrm{V}$ is utterance-final), and fail to align the right edge of the miminal NP consisting only of $\mathrm{N}$ (since doing so would entail violating BINMIN). The adjunct non-cohering candidate (a), in failing to align the right edge of AP with the right edge of a $\phi$, incurs one more violation than (b), which maps the entire subject to a $\phi$. Thus, if ALIGN-R is allowed to decide the outcome, candidate (b) emerges as optimal, correctly predicting the domains of High Tone Deletion in Kinyambo. Conversely, if ALIGN-L dominates Align-R, as in (24), the adjunct non-cohering candidate is optimal. Both candidates fail to align the left edge of $v \mathrm{P}$ with the left edge of a $\phi$, and succeed in aligning the left edge of NP with the left edge of a $\phi$, but candidate (a) aligns the left edge of AP with the left edge of a $\phi$ while candidate (b) does not.

Note that even if a real language with adjunct non-cohesion were found, bearing out the ANCP and revealing that typologies containing adjunct non-cohesion are not overgenerating, nonetheless all the Align/Wrap systems in this experiment would still have a problem with undergeneration, since none of them generate the correct phrasings for even these four sentences of Kinyambo. (This is not to say that no Align system can ever produce Kinyambo, of course; the typology can always be expanded by adding more constraints, or altering various assumptions.) Match systems, by contrast, have no undergeneration problem (for this fragment of Kinyambo), so long as the highest segment of a maximal projection is visible. Generally speaking, Match systems' typologies are smaller than the typologies of the corresponding Align systems, while still achieving better coverage of the Kinyambo facts than the Align systems.

\section{Conclusion}

In conclusion, we have shown that the highest segment of a maximal projection must be visible to the syntax-prosody mapping constraints in order to ensure that adjuncts prosodically cohere to their syntactic hosts. Furthermore, we have demonstrated that Match Theory, in our particular implementation of it, is capable of accounting for certain phrasing data from Kinyambo, including data involving the branchingness effect identified by Bickmore $(1989,1990)$. While the Align/Wrap systems we tested are less successful in this regard, it should be noted that they employ a nonstandard assumption in that they do not disregard functional projections, and further research is needed to determine their predictions given more standard assumptions.

In Match systems, the branchingness effect arises due to an interplay of binarity constraints, with BINMAX outranking BINMIN, which in turn outranks MATCH(XP, $\phi)$. Interestingly, it does not matter for our purposes here whether binarity is counted in terms of a prosodic node's children, or in terms of the number of words it dominates. Research on additional binarity-driven phrasing phenomena is needed to determine the full effects of the different families of binarity constraints under consideration. 


\section{References}

Alber, Birgit, Natalie DelBusso, \& Alan Prince. 2015. From Intensional Properties to Universal Support. ROA-1235.

Bellik, Jenny, Ozan Bellik, \& Nick Kalivoda. 2015. Syntax-Prosody in OT. JavaScript application: https : / / github.com/syntax-prosody-ot/main

Bickmore, Lee. 1989. Kinyambo Prosody. Ph.D. thesis, UCLA.

Bickmore, Lee. 1990. Branching Nodes and Prosodic Categories. In Sharon Inkelas \& Draga Zec (eds.), The Phonology-Syntax Connection.

Chomsky, Noam. 1959. On Certain Formal Properties of Grammars. Information and Control 2, pp. 137167.

Chomsky, Noam. 1986. Barriers. Cambridge, MA: MIT Press.

Chomsky, Noam. 1995. Bare Phrase Structure. In Gert Webelhuth (ed.), Government and Binding Theory and the Minimalist Program, pp. 385-439. Wiley-Blackwell.

Elfner, Emily. 2012. Syntax-Prosody Interaction in Irish. Ph.D. thesis, UMass Amherst.

Ito, Junko \& Armin Mester. 2013. Prosodic subcategories in Japanese. Lingua 124, pp. 20-40.

May, Robert. 1985. Logical Form: Its Structure and Derivation. Cambridge, MA: MIT Press.

McCarthy, John \& Alan Prince. Generalized alignment. Linguistics Department Faculty Publication Series. Paper 12. http://scholarworks.umass.edu/linguist_faculty_pubs/12

Myrberg, Sara. 2013. Sisterhood in prosodic branching. Phonology, Vol. 30, No. 1, pp. 73-124.

Nespor, Marina \& Irene Vogel. 1986. Prosodic Phonology.

Prince, Alan, Bruce Tesar, \& Nazarré Merchant. 2015. OTWorkplace. http://sites.google.com/ site/otworkplace/

Selkirk, Elisabeth. 1986. On derived domains in sentence phonology. Phonology Yearbook, Vol. 3, pp. 371405.

Selkirk, Elisabeth. 2011. The Syntax-Phonology Interface. In John A. Goldsmith, Jason Riggle, \& Alan C. L. Yu (eds.), The Handbook of Phonological Theory, 2nd edition. Wiley-Blackwell.

Truckenbrodt, Hubert. 1995. Phonological Phrases: Their Relation to Syntax, Focus, and Prominence. Ph.D. thesis, MIT.

Truckenbrodt, Hubert. 1999. On the Relation between Syntactic Phrases and Phonological Phrases. Linguistic Inquiry, Vol. 30, No. 2, pp. 219-255. 